\title{
Interface energy and its influence on interface fracture between metal and ceramic thin films in nanoscale
}

\author{
L. H. Liang, ${ }^{\text {a) }}$ X. M. You, H. S. Ma, and Y. G. Wei \\ LNM, Institute of Mechanics, Chinese Academy of Sciences, Beijing 100190, China
}

(Received 29 July 2010; accepted 12 September 2010; published online 27 October 2010)

\begin{abstract}
A theoretical model about the size-dependent interface energy between two thin films with different materials is developed by considering the chemical bonding contribution based on the thermodynamic expressions and the structure strain contribution based on the mechanical characteristics. The interface energy decreases with reducing thickness of thin films, and is determined by such available thermodynamic and mechanical parameters as the melting entropy, the melting enthalpy, the shear modulus of two materials, etc. The predicted interface energies of some metal $/ \mathrm{MgO}$ and metal $/ \mathrm{Al}_{2} \mathrm{O}_{3}$ interfaces based on the model are consistent with the results based on the molecular mechanics calculation. Furthermore, the interface fracture properties of $\mathrm{Ag} / \mathrm{MgO}$ and $\mathrm{Ni} / \mathrm{Al}_{2} \mathrm{O}_{3}$ based on the atomistic simulation are further compared with each other. The fracture strength and the toughness of the interface with the smaller structure interface energy are both found to be lower. The intrinsic relations among the interface energy, the interface strength, and the fracture toughness are discussed by introducing the related interface potential and the interface stress. The microscopic interface fracture toughness is found to equal the structure interface energy in nanoscale, and the microscopic fracture strength is proportional to the fracture toughness. (C) 2010 American Institute of Physics. [doi:10.1063/1.3501090]
\end{abstract}

\section{INTRODUCTION}

The study on interface energy has attracted great attention in both experimental and theoretical aspects due to its importance in extensive fields. ${ }^{1-4}$ The stability of interfaces determined by interface energy affects the performance of structures and devices. Specially, the interfaces between metals and ceramics play important role in thermal barrier coatings used in aircraft and power generation turbines, and they have also extensive applications in catalytic converters, field effect transistors, anticorrosion coatings, etc. The failure of metal/ceramic interfaces may lead to the breakdown of these structures and devices. The fracture is a kind of severe interface failure mode, the fracture strength, and the fracture toughness are important parameters determining the interface fracture properties and are closely related to the interface energy. The experimental measurement of interface energy is difficult and lacking. The theoretical calculation based on the first principle, the embedded atomic method, and the molecular dynamics is complicated and time-consuming. Therefore, the scientific theoretical prediction of the interface energy and the further understanding of its influence on the interface fracture are significant. The size effect of interface energy cannot be neglected with the application of lowdimensional materials and the development of microelectromechanical systems and nanoelectromechanical systems. Study has found that the interface energy is size-dependent, ${ }^{1-3}$ and the size effect affects the deformation and the phase transition of nanomaterials and the stability of interfaces.

In this paper, a simple theoretical model on the size-

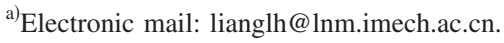

dependent interface energy between heterogeneous materials is developed including the chemical intrinsic interface energy and the structure interface energy, the interface energies of some metal/ceramic systems are predicted based on the model. Furthermore, the interface energy and the interface fracture strength of $\mathrm{Ag} / \mathrm{MgO}$ and $\mathrm{Ni} / \mathrm{Al}_{2} \mathrm{O}_{3}$ systems are compared with each other, and the relation between two interface quantities is discussed. Moreover, the microscopic interface fracture toughness is determined by the structure interface energy in nanoscale based on the interface potential discussion.

\section{MODEL}

The interface energy $\gamma$ is the excess energy per unit area of a system due to appearing of the interface, and it is originated from the change in the interfacial atomic chemical bonding and the structure strain at the interface, i.e., $\gamma$ includes the chemical interface energy $\gamma_{c}$ and the structural interface energy $\gamma_{s}$. The chemical interface energy is larger for metal/ceramic interfaces due to the greater chemical bonding difference of two materials, and it is related to the intrinsic solid-solid interface energies of two materials $\gamma_{c 1}$ and $\gamma_{c 2}$, respectively, thus $\gamma_{c}$ is taken as the average value of $\gamma_{c 1}$ and $\gamma_{c 2}$ approximately. The size effect of the interface energy should be considered, which becomes obvious in nanoscale. The size-dependent intrinsic solid-solid interface energy $\gamma_{c i}$ of single materials is first considered, $i=1,2$ denotes two materials, respectively, and the subscripts $i$ and $c$ are elliptical in subsequent part for the simplicity of the expression.

Let $\gamma_{b}$ be the solid-solid interface energy of single bulk materials, and $\Delta \gamma=\gamma-\gamma_{b}$ be the change in the interface energy in nanoscale compared to the bulk. The bulk solid-solid 
interface energy $\gamma_{b}$ is about two times of the solid-liquid interface energy $\gamma_{m}$ of the corresponding materials. ${ }^{5}$ The solid-liquid interface energy $\gamma_{m}=2 h S_{v} H_{m} /\left(3 V_{m} R\right)$ based on Gibbs-Thomson equation, where $h$ is the atomic diameter of the materials, $S_{v}$ is the vibrational part of the melting entropy $S_{m}=H_{m} / T_{m}, H_{m}$ is the melting enthalpy, $T_{m}$ is the melting temperature, $V_{m}$ is the molar volume of the solids, and $R$ is the ideal gas constant. ${ }^{6}$ Therefore, the bulk solid-solid interface energy can be expressed as

$$
\gamma_{b}=4 h S_{v} H_{m} /\left(3 V_{m} R\right)
$$

On the other hand, the interface energy is related to the interface stress, according to the thermodynamic definition of the interface stress $f,{ }^{7,8} f=\partial G / \partial A=\partial(\gamma A) / \partial A=\gamma+A \partial \gamma / \partial A$ $\approx \gamma+A \Delta \gamma / \Delta A$, where $G$ is the interface excess Gibbs free energy and $A$ is the interface area. The above equation can be rearranged as $\Delta \gamma=(f-\gamma) \Delta A / A, \quad$ therefore, $\quad \gamma-\gamma_{b}=(f$ $-\gamma) \Delta A / A$ and the size-dependent interface energy can be expressed as

$$
\gamma=\left(f \Delta A / A+\gamma_{b}\right) /(1+\Delta A / A)
$$

i.e., the size effect is related to the change in the interface area. Under the small strain, $\Delta A /(2 A)=\Delta V /(3 V)$, where $V$ is the volume of the solid with the interface area $A$. Combining with the definition of the compressibility $\kappa, \kappa=-\Delta V /(V P)$, where $P$ is the pressure difference between the inside and the outside of the solid, $\Delta A / A=-2 \kappa P / 3$. According to the Laplace-Young equation, for a compressible spherical particle with diameter $D$ immersed in the liquid, $P=2 f A /(3 V){ }^{8}$ $A / V=6 / D$ for the solid-liquid interface. For the solid-solid interface discussed here, $A / V=3 / D$ considering that two solid-liquid interfaces of particles combine to form one grain boundary or solid-solid interface, thus $P=2 f / D$, the result is reasonable since the elastic modulus of the solid is larger than that of the liquid and the pressure difference of solidsolid is smaller than that of solid-liquid. Therefore, $\Delta A / A$ $=-4 \kappa f /(3 D)$, and the size-dependent solid-solid interface energy can be expressed as

$$
\gamma=\gamma_{b}\left(1-\frac{4 \kappa f^{2}}{3 \gamma_{b} D}\right) /\left(1-\frac{4 \kappa f}{3 D}\right)
$$

The expression of the intrinsic interface stress $f$ has been obtained as ${ }^{2,9}$

$$
f=-\left[\left(3 \gamma_{m} D_{0}\right) /(8 \kappa)\right]^{1 / 2}
$$

based on the similar consideration to the above discussion, where the negative sign shows the compressive interface stress, $D_{0}$ is the critical size, $D_{0}=3 h, 2 h$ for particles and thin films, respectively. ${ }^{9}$ Substituting Eq. (4) into Eq. (3), $\gamma$ $=\gamma_{b}\left[1-D_{0} /(4 D)\right] /\left[1-\gamma_{b} D_{0} /(4 f D)\right]$. Considering the interface stress $f$ is one order larger than the interface energy $\gamma_{b}$, the denominator in the above equation can be neglected.

Now the subscripts $i$ and $c$ are retrieved, the sizedependent intrinsic solid-solid interface energy $\gamma_{c i}$ of single materials can be expressed as

$$
\gamma_{c i}=\gamma_{b i}\left(1-\frac{D_{0 i}}{4 D}\right)
$$

where $D$ is the diameter and the thickness for particles and thin films, respectively. Equation (5) is consistent with the quantum chemical calculation that surface energy is inversely proportional to the reciprocal size of a particle. ${ }^{10}$ For interfaces between metal and ceramic thin films, $D=t_{1}+t_{2}$ denotes the total thickness, $t_{1}$ and $t_{2}$ are the thickness of the metal and the ceramic thin films, respectively. Considering the average of two materials and substituting Eq. (1) into Eq. (5), the size-dependent chemical interface energy between two materials can be expressed as

$$
\gamma_{c}=\frac{2}{3 R}\left[\frac{h_{1} S_{v 1} H_{m 1}}{V_{m 1}}\left(1-\frac{h_{1}}{2 D}\right)+\frac{h_{2} S_{v 2} H_{m 2}}{V_{m 2}}\left(1-\frac{h_{2}}{2 D}\right)\right] .
$$

Equation (6) shows that the chemical interface energy decreases with reducing thickness of thin films depending on some available bulk thermodynamic parameters of two materials such as the melting entropy and enthalpy.

On the other hand, the structure interface energy $\gamma_{s}$ caused by the lattice mismatch between two materials and the dislocation strain at the interface should be considered. According to the Matthews model, ${ }^{11}$ the initial interface energy $\gamma_{0}$ for a fully relaxed thin film on a substrate can be expressed as $\gamma_{0}=C b \varepsilon_{0}\{\ln [D /(2 b)]+1\} /(2 \pi)$, where $C$ $=\left\{2\left[\left(1-\nu_{1}\right) / G_{1}+\left(1-\nu_{2}\right) / G_{2}\right]\right\}^{-1}$ is the effective elastic modulus, $\nu$ is the Poisson's ratio, and $G$ is the shear modulus, $b=\left(h_{1}+h_{2}\right) / 2$ is the Burgers vector, $\varepsilon_{0}=\left(h_{2}-h_{1}\right) / h_{1}$ is the misfit strain with $h_{2}>h_{1}$. D/2 represents the radius of the effective dislocation stress-field. Considering the semicoherent interfaces between two materials, the coherency strain $\varepsilon$ meets partial lattice matching, and generally, the major contribution to the structure interface energy is the energy associated with the formation of dislocations that accommodates part of the misfit, ${ }^{12}$ thus $\gamma_{s}$ is proportional to the dislocation strain $\left(\varepsilon_{0}-\varepsilon\right)$. Therefore, the structure interface energy can be expressed as $\gamma_{s}=\gamma_{0}\left(1-\varepsilon / \varepsilon_{0}\right)$, where $\varepsilon$ is also sizedependent due to the intrinsic lattice strain of thin films compared to the corresponding bulk materials and it is determined by

$$
\varepsilon=-\frac{2 \kappa f}{3 D}
$$

based on the Laplace-Young equation and the isotropic assumption. $^{2,9}$ The compressibility $\kappa=1 / B$, where $B$ is the volume modulus and taken as the average value of two materials. The intrinsic interface stress $f$ refers to Eq. (4), $\gamma_{m}$ and $D_{0}$ are also taken as the average values of two materials, respectively. Finally, the structure interface energy between two materials can be expressed as 
TABLE I. The interface energies $\left(\mathrm{J} / \mathrm{m}^{2}\right)$ of some metal/ceramic systems based on Eq. (9) and the molecular mechanics (MM) calculation (Refs. 13-15). In Eq. (9), taking $D=20 \mathrm{~nm}$, the other parameters are in Table II.

\begin{tabular}{lccccccc}
\hline \hline & $\mathrm{Ni} / \mathrm{MgO}$ & $\mathrm{Pd} / \mathrm{MgO}$ & $\mathrm{Ag} / \mathrm{MgO}$ & $\mathrm{Au} / \mathrm{MgO}$ & $\mathrm{Cu} / \mathrm{Al}_{2} \mathrm{O}_{3}$ & $\mathrm{Ag}_{/} \mathrm{Al}_{2} \mathrm{O}_{3}$ & $\mathrm{Au} / \mathrm{Al}_{2} \mathrm{O}_{3}$ \\
\hline Eq. (9) & 4.6 & 3.59 & 3 & 3.1 & 3.26 & 2.47 & 2.5 \\
$\mathrm{MM}$ & 4.6 (Ref. 13) & 3.84 (Ref. 4) & 4 (Ref. 14) & 3.68 (Ref. 14) & 3.24 (Ref. 15) & 2.31 (Ref. 15) & 2.9 (Ref. 15) \\
\hline \hline
\end{tabular}

$$
\begin{aligned}
\gamma_{s}= & \frac{G_{1} G_{2} b \varepsilon_{0}}{4 \pi\left[\left(1-v_{1}\right) G_{2}+\left(1-v_{2}\right) G_{1}\right]}\left[\ln \left(\frac{D}{2 b}\right)+1\right] \\
& \times\left(1-\frac{\varepsilon}{\varepsilon_{0}}\right) .
\end{aligned}
$$

Substituting Eq. (7) into Eq. (8), the size-dependent structural interface energy can be obtained by using of some available bulk mechanical parameters of two materials such as the shear modulus and the Poisson's ratio. $\gamma_{s}$ also decreases with reducing thickness of thin films. Combining Eqs. (6) and (8), the total interface energy $\gamma$ is expressed as

$$
\gamma=\gamma_{c}+\gamma_{s}
$$

\section{RESULTS AND DISCUSSIONS}

We calculated the interface energies of some metal/ ceramic interfaces, such as $\mathrm{Ni} / \mathrm{MgO}, \mathrm{Ag} / \mathrm{MgO}, \mathrm{Ag} / \mathrm{Al}_{2} \mathrm{O}_{3}$, etc. in terms of Eq. (9), the results are in Table I. It can be found that the predicted values based on our model are in agreement with the calculated interface energies based on the molecular mechanics. ${ }^{13-15}$ The interface energy keeps several joules per meter square despite of size effect. Figure 1 shows the predicted interface energy of $\mathrm{Ag} / \mathrm{MgO}$ and $\mathrm{Ni} / \mathrm{Al}_{2} \mathrm{O}_{3}$ systems in the same thickness range based on the model by use of some available thermodynamic parameters. ${ }^{16-24}$ It can be seen from the figure that the interface energy decreases with reducing thickness of thin films. We also calculate the interface energy of $\mathrm{Ag} / \mathrm{Ni}$ and $\mathrm{Ni} / \mathrm{Co}$ interfaces, the interface energy of metal/metal interfaces is smaller than that of metal/ ceramic interfaces. The chemical interface energy of $\mathrm{Ag} / \mathrm{Ni}$ is about $0.84 \mathrm{~J} / \mathrm{m}^{2}$ at the interface thickness of $20 \mathrm{~nm}$, which is very close to the experimental results of $0.76 \mathrm{~J} / \mathrm{m}^{2}{ }^{25}$ The decrease in the chemical interface energy in nanoscale can explain the observed interfacial phase segregation phenomena in nanoscale $\mathrm{Ni} / \mathrm{Co}$ systems. $\mathrm{Ni}$ and $\mathrm{Co}$ are miscible elements and can form alloy in bulk materials but in nanostructured Ni/Co systems they did not form the alloy as expected in their bulk phase diagram, ${ }^{26}$ which is because that the stability of the interface increases with the decrease in the chemical interface energy and the interface may exist stably. Note that the total interface energy $\gamma$ of $\mathrm{Ag} / \mathrm{MgO}$ is smaller than that of $\mathrm{Ni} / \mathrm{Al}_{2} \mathrm{O}_{3}$ at the same size [see Fig. 1(a)]. However, the chemical interface energy $\gamma_{c}$ of $\mathrm{Ag} / \mathrm{MgO}$ is slightly larger than that of $\mathrm{Ni} / \mathrm{Al}_{2} \mathrm{O}_{3}$ [Fig. 1(b)], which implies that the interface stability of $\mathrm{Ag} / \mathrm{MgO}$ is weaker and the interface may separate more easily. While the structure interface energy $\gamma_{s}$ of $\mathrm{Ag} / \mathrm{MgO}$ is smaller than that of $\mathrm{Ni} / \mathrm{Al}_{2} \mathrm{O}_{3}$, i.e., the structural strain contribution to the total interface energy is larger for $\mathrm{Ni} / \mathrm{Al}_{2} \mathrm{O}_{3}$, which may imply that the larger strain work needs to be overcome to separate the interface.

Figure 2 shows the interface tension separation simulation of $\mathrm{Ag} / \mathrm{MgO}$ and $\mathrm{Ni} / \mathrm{Al}_{2} \mathrm{O}_{3}$ systems based on the molecular mechanics calculation ${ }^{27}$ and the first principle calculation, ${ }^{28}$ respectively. It can be seen that two stressdisplacement curves show the same shape despite of different simulation methods, and the interface tension stress $\sigma$ increases first with increasing interface tension displacement $\Delta x$, then decreases after reaching the maximum stress, i.e., the interface fracture strength $\sigma_{b}$, vanishes finally when the interface separates, the trend is consistent with the cohesive zone model describing the interface fracture. ${ }^{29}$ Differently, the interface fracture strength $\sigma_{b}$ of $\mathrm{Ag} / \mathrm{MgO}$ is lower than that of $\mathrm{Ni} / \mathrm{Al}_{2} \mathrm{O}_{3}$, and the fracture toughness $\Gamma_{0}$, i.e., the area under the $\sigma-\Delta x$ curve, of the former is also smaller than that of the latter. Note that the fracture strength and toughness of $\mathrm{Ag} / \mathrm{MgO}$ interface with the larger chemical interface energy are both smaller, i.e., the interface fractures more easily, which reflects that the stability of the interface with the larger chemical interface energy is really weaker. The smaller fracture toughness also indicates that the smaller work needs to be overcome in the fracture process, which may be related to the weaker interface adhesion ability of $\mathrm{Ag} / \mathrm{MgO}$, while the interface possess the smaller structural interface energy at the same time.

In order to further understand the intrinsic relation between the interface energy and the interface fracture properties, we calculate the interface potential energy $\phi$ and the
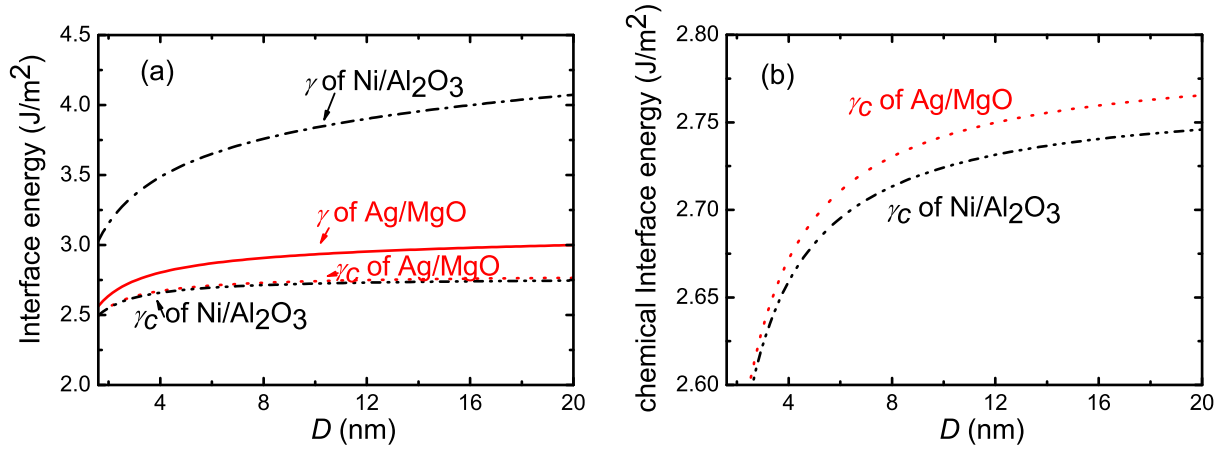

FIG. 1. (Color online) The total interface energy (a) and the chemical interface energy (b) vs the total thickness of metal/ceramic interfaces for $\mathrm{Ag} /$ $\mathrm{MgO}$ and $\mathrm{Ni} / \mathrm{Al}_{2} \mathrm{O}_{3}$ in terms of Eqs. (6) and (9), respectively. The related parameters in the equations are in Table II. 


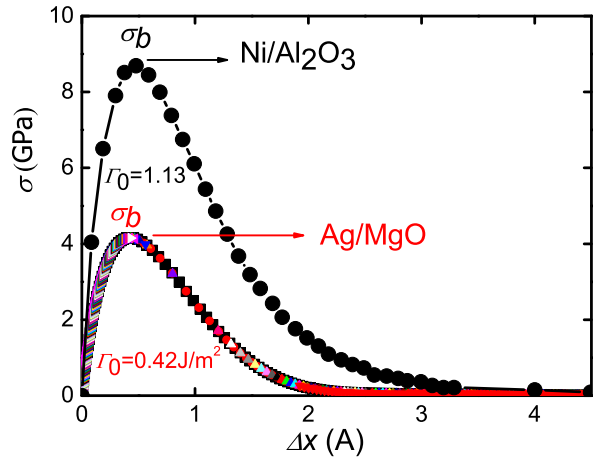

FIG. 2. (Color online) The interface tension stress vs the displacement of $\mathrm{Ag} / \mathrm{MgO}$ and $\mathrm{Ni} / \mathrm{Al}_{2} \mathrm{O}_{3}$ based on the molecular mechanics (Ref. 27) and the first principle calculations (Ref. 28), respectively.

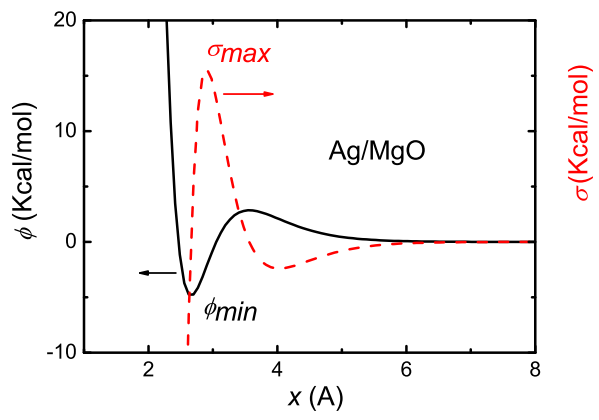

FIG. 3. (Color online) The interface potential and the stress vs the interface distance of $\mathrm{Ag} / \mathrm{MgO}$ in terms of Eq. (10). The potential parameters are in Table I in Ref. 30.
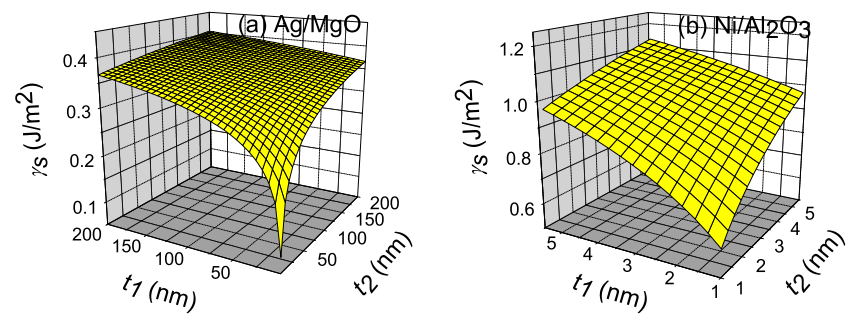

FIG. 4. (Color online) The structure interface energy vs the respective thickness of the metal and the ceramic thin films (a) for $\mathrm{Ag} / \mathrm{MgO}$ and (b) for $\mathrm{Ni} / \mathrm{Al}_{2} \mathrm{O}_{3}$ in terms of Eq. (8) with $D=t_{1}+t_{2}$. The related parameters in the equations are in Table II. corresponding interface stress of $\mathrm{Ag} / \mathrm{MgO}$ system. For metal/ ceramic interfaces, $\phi$ refers to the modified RahmanStillinger-Lemberg potential as follows

$$
\phi=a_{0} e^{d\left(1-\frac{x}{c_{0}}\right)}+\frac{a_{1}}{1+e^{b_{1}\left(x-c_{1}\right)}}+\frac{a_{2}}{1+e^{b_{2}\left(x-c_{2}\right)}}+\frac{a_{3}}{1+e^{b_{3}\left(x-c_{3}\right)}},
$$

where $x$ is the interface atomic distance, the other parameters are the potential parameters given by Chen-Mobius inversion method based on the ab initio calculation. ${ }^{30}$ Considering two kinds of atomic pairs $\mathrm{Ag} / \mathrm{Mg}$ and $\mathrm{Ag} / \mathrm{O}$ across the interface, the potential parameters are all approximately taken as the average values of the both for the simplicity of the calculation. The interface stress $\sigma=\partial \phi / \partial x$. Figure 3 shows the calculated interface potential of $\mathrm{Ag} / \mathrm{MgO}$ based on Eq. (10), the absolute value of the minimum potential energy $\left|\phi_{\min }\right|$ or the interface adhesive energy is $4.77 \mathrm{kcal} / \mathrm{mol}$, i.e., $1.77 \mathrm{GPa}$ after the unit conversion, that is $0.47 \mathrm{~J} / \mathrm{m}^{2}$ after multiplying by the corresponding interface distance. $\left|\phi_{\min }\right|$ almost agrees with the microscopic interface fracture toughness $\Gamma_{0}$ of $\mathrm{Ag}$ / $\mathrm{MgO}\left(0.42 \mathrm{~J} / \mathrm{m}^{2}\right)$ based on our molecular mechanics simulation as shown in Fig. 2, and also roughly agrees with the calculated structural interface energy $\gamma_{s}, 0.41 \mathrm{~J} / \mathrm{m}^{2}$ as shown in Fig. 4(a), based on our analytical model when the thickness of $\mathrm{Ag}$ and $\mathrm{MgO}$ thin films are both $200 \mathrm{~nm}$. For $\mathrm{Ni} / \mathrm{Al}_{2} \mathrm{O}_{3}, \Gamma_{0}$ is $1.13 \mathrm{~J} / \mathrm{m}^{2}$ (Fig. 2), which also agrees with $\gamma_{s}$ at $D=11 \mathrm{~nm}$ as shown in Fig. 4(b) corresponding to the scale of the first principle calculation. Therefore, the structural interface energy in nanoscale corresponds to the microscopic interface fracture toughness or the interface adhesive work. Moreover, the larger $\gamma_{s}$ or the fracture toughness is, the higher is the strength, since that the larger $\left|\phi_{\min }\right|$ is, the higher the maximum stress $\sigma_{\max }$ is, the result is in agreement with the previous discussion. ${ }^{31}$

Figure 3 also shows the interface stress of $\mathrm{Ag} / \mathrm{MgO}$, it can be seen that the maximum stress $\sigma_{\text {max }}$, i.e., the fracture strength, is $15.44 \mathrm{Kcal} / \mathrm{mol}$, that is $5.7 \mathrm{GPa}$ after the unit conversion, which is close to the calculation result based on the our molecular mechanics as shown in Fig. 2. In fact, the calculation in Fig. 2 is based on the same interface potential, but is more accurate. On the other hand, the structure interface energy is much smaller than the chemical interface energy, e.g., $\gamma_{c}$ is $3.16 \mathrm{~J} / \mathrm{m}^{2}$, more than one order of $\gamma_{s}$ $\left(0.41 \mathrm{~J} / \mathrm{m}^{2}\right)$ for $\mathrm{Ag} / \mathrm{MgO}$ at the same thickness. The chemi-

TABLE II. The related parameters in the equations. $h=2 r$ with the atomic radius $r . h$ is taken as the average value of both elements for MgO, and taken as the bond length for $\mathrm{Al}_{2} \mathrm{O}_{3}$. For ceramics, $V=M / \rho$ with the molar mass $M$ and the density $\rho, B=2 G(1+\nu) /[3(1-2 \nu)]$. For metals, $S_{v}=S_{m}$, for ceramic semiconductors, $S_{v}=0.4 S_{m}$ (Ref. 16).

\begin{tabular}{lccccccc}
\hline \hline & $\mathrm{Ag}$ & $\mathrm{Au}$ & $\mathrm{Ni}$ & $\mathrm{Pd}$ & $\mathrm{Cu}$ & $\mathrm{MgO}$ & $\mathrm{Al}_{2} \mathrm{O}_{3}$ \\
\hline$h(\mathrm{~nm})($ Ref. 17) & 0.3194 & 0.3188 & 0.2754 & 0.304 & 0.2806 & 0.3376 (Ref. 17) & $0.324($ Ref. 23) \\
$H_{m}(\mathrm{~kJ} / \mathrm{mol})($ Ref. 18) & 11.3 & 12.55 & 17.47 & 17.6 & 13.05 & 90 (Ref. 20) & $111.4($ Ref. 24) \\
$T_{m}(\mathrm{~K})($ Ref. 18) & 1234 & 1337.58 & 1726 & 1825 & 1357.6 & 3073 (Ref. 21) & $2326($ Ref. 24) \\
$S_{v}(\mathrm{~J} / \mathrm{mol} / \mathrm{K})($ Ref. 18) & 9.157 & 9.383 & 10.122 & 9.644 & 9.613 & 11.7 (Refs. 20 and 21) & $19.157($ Ref. 24) \\
$V_{m}\left(\mathrm{~cm}^{3} / \mathrm{mol}\right)($ Ref. 18) & 10.3 & 10.2 & 6.59 & 8.9 & 7.1 & 11.26 (Ref. 21) & $25.49($ Ref. 24) \\
$G(\mathrm{GPa})($ Ref. 19) & 30.3 & 26 & 76 & 43.6 & 48.3 & 130 (Ref. 22) & $150($ Ref. 24) \\
$\nu(\mathrm{Ref} .19)$ & 0.367 & 0.42 & 0.312 & 0.39 & 0.343 & 0.18 (Ref. 22) & $0.22($ Ref. 24) \\
$B(\mathrm{GPa})($ Ref. 19) & 103.6 & 171 & 177.3 & 187 & 137.8 & 160 (Ref. 22) & $217.86($ Ref. 24) \\
\hline \hline
\end{tabular}


cal interface energy should also be related to the total fracture energy, and may contribute to the plastic dissipation, how it affects the fracture behavior has yet to be discovered.

\section{CONCLUSION}

In summary, a theoretical model about the size dependence of the interface energy between two thin films with different materials is developed. The interface energy decreases as the thickness of thin films decreases, and the size effect depends on the atomic diameter, the melting enthalpy, the shear modulus of two materials, etc. The theoretically predicted interface energy is in agreement with the results based on the molecular mechanics calculation. The comparison of the interface energy and the interface fracture strength of $\mathrm{Ag} / \mathrm{MgO}$ and $\mathrm{Ni} / \mathrm{Al}_{2} \mathrm{O}_{3}$ systems indicates that the fracture strength is higher for the interface with the larger structure interface energy, the former corresponds to the maximum of the first order derivative of the interface potential, and the latter corresponds to the microscopic interface fracture toughness.

\section{ACKNOWLEDGMENTS}

The work is supported by the Research Grants from NSFC through Grant Nos. 10802088, 10832008, and 10702070 and the LNM initial funding for young investigators through Grant Nos. 10721202 and 10932011.

${ }^{1}$ J. Wang, D. Wolf, S. R. Philpot, and H. Gleiter, Philos. Mag. A 73, 517 (1996).

${ }^{2}$ Q. Jiang, D. S. Zhao, and M. Zhao, Acta Mater. 49, 3143 (2001).

${ }^{3}$ G. Ouyang, L. H. Liang, C. X. Wang, and G. W. Yang, Appl. Phys. Lett. 88, 091914 (2006).

${ }^{4}$ M. Zhao, W. T. Zheng, J. C. Li, Z. Wen, M. X. Gu, and C. Q. Sun, Phys. Rev. B 75, 085427 (2007).
${ }^{5}$ A. Kotzé and D. Kuhlmann-Wilsdorf, Appl. Phys. Lett. 9, 96 (1966).

${ }^{6}$ Q. Jiang, H. X. Shi, and M. Zhao, Acta Mater. 47, 2109 (1999).

${ }^{7}$ R. R. Vanfleet and J. M. Mochel, Surf. Sci. 341, 40 (1995).

${ }^{8}$ J. Weissmüller and J. W. Cahn, Acta Mater. 45, 1899 (1997).

${ }^{9}$ Q. Jiang, L. H. Liang, and D. S. Zhao, J. Phys. Chem. B 105, 6275 (2001).

${ }^{10}$ H. Müller, C. Opitz, K. Strickert, and L. Skala, Z. Phys. Chem. (Leipzig) 268, 634 (1987).

${ }^{11}$ J. W. Matthews, in Epitaxial Growth, edited by J. W. Matthews (Academic, New York, 1975), p. 559.

${ }^{12}$ R. C. Cammarata, K. Sieradzki, and F. Spaepen, J. Appl. Phys. 87, 1227 (2000).

${ }^{13}$ Y. Long and N. X. Chen, Comput. Mater. Sci. 42, 426 (2008).

${ }^{14}$ Y. Long and N. X. Chen, Surf. Sci. 602, 1122 (2008).

${ }^{15}$ Y. Long and N. X. Chen, J. Phys.: Condens. Matter 21, 315003 (2009).

${ }^{16}$ A. R. Regel' and V. M. Glazov, Semiconductors 29, 405 (1995).

${ }^{17}$ H. W. King, in Physical Metallurgy, edited by R. W. Cahn (North-Holland, Amsterdam, 1983), p. 64.

${ }^{18}$ Periodic Table of the Elements (Sargent-Welch Scientific, Skokie, Illinois, 1980), p. 1

${ }^{19}$ Smithells Metals Reference Book, edited by E. A. Brandes (Butterworths, London, 1983), pp. 15-22.

${ }^{20}$ R. E. Cohen and J. S. Weitz, in Properties of Earth and Planetary Materials at High Temperature, edited by M. H. Manghnani and T. Yagi (AMER Geophysical Union, Washington, DC, 1998), Vol. 101, p. 185.

${ }^{21}$ http://en.wikipedia.org/wiki/Magnesium_oxide

${ }^{22}$ C. S. Zha, H. K. Mao, and R. J. Hemley, Proc. Natl. Acad. Sci. U.S.A. 97, 13494 (2000).

${ }^{23} \mathrm{http} / / / \mathrm{www} . q i v x . c o m / i s p t / e l e m e n t s / p t w \_013 . p h p$

${ }^{24}$ http://www.matweb.com/search/DataSheet.aspx?MatGUID $=\mathrm{c} 8 \mathrm{c} 56 \mathrm{ad} 547 \mathrm{ae} 4 \mathrm{cfabad} 15977 \mathrm{bfb} 537 \mathrm{f} 1$

${ }^{25}$ D. Josell and F. Spaepen, Acta Metall. Mater. 41, 3017 (1993).

${ }^{26}$ G. M. Chow, W. C. Goh, Y. K. Hwu, T. S. Cho, J. H. Je, H. H. Lee, H. C. Kang, D. Y. Noh, C. K. Lin, and W. D. Chang, Appl. Phys. Lett. 75, 2503 (1999).

${ }^{27}$ X. M. You, L. H. Liang, H. S. Ma, and Y. G. Wei, private communications.

${ }^{28}$ Y. Jiang, Y. G. Wei, J. R. Smith, J. W. Hutchinson, and A. G. Evans, Int. J. Mater. Res. 101, 8 (2010).

${ }^{29}$ H. F. Zhao and Y. G. Wei, Int. J. Fract. 144, 103 (2007).

${ }^{30}$ Y. Long, N. X. Chen, and W. Q. Zhang, J. Phys.: Condens. Matter 17, 2045 (2005).

${ }^{31}$ Y. G. Wei and J. W. Hutchinson, Philos. Mag. 88, 3841 (2008). 